\title{
Protected Object Models as the Basis of Distance Measuring Units Algorithms Synthesis
}

\author{
Viktorija Aditaja ${ }^{1}$, Tatjana Lomane ${ }^{2 *}$ \\ ${ }^{1-2}$ Riga Technical University
}

\begin{abstract}
In the present study, the algorithms of distance protection (DP) for a high-voltage transmission line are considered. The single, parallel and tapped transformer lines can be as a protected object. The steady-state and transient modes in the model of protected objects are described by the node-voltage equations, which may be in simplified record either taking into account all parameters or not taking into account some mode parameters. DP efficiency depends on the record form of the nodevoltage equations. The programmed distance measuring units algorithms synthesized based on the protected object model are analysed.
\end{abstract}

Keywords - Distance measuring units, efficiency of operation, HVTL model, node voltage equation.

\section{INTRODUCTION}

With the development and complexity of electrical networks, the regimes of weighting electric power systems (EPS) and the desire to improve the power systems reliability, the demands for effectiveness of relay protection become topical [1]-[4]. The key factors of relay protection reliability for providing the uninterrupted power supply are sensitivity, selectivity, and speed. The fulfillment of these requirements to a large extent are connected with the effectiveness of its measuring units. The development trends of microprocessor (MP) technology and the distance protection principle allow solving the tasks of increasing the efficiency of distance protection (DP) operation by the use of more complex models of the protected object for the programmed distance measuring units (PDMU) algorithms synthesis.

One of the ways to solve the problem of the effectiveness increase is creating the PDMU algorithms with taking into account EPS operation modes, parameters, and network configurations [2]-[13]. The paper discusses PDMU algorithms, based on the specified models of the protected object. The effectiveness of PDMU operation is analyzed in this paper.

\section{Mathematical Model of CONTROL OBJeCt}

Distance measuring units are devices that determine impedance to fault location by the measured values of currents and voltages at the point of protective relay installation. The impedance calculation algorithm depends primarily on the mathematical model of the protected object operation underlying the synthesis. The controlled object is the high- voltage transmission line, which model depends on the voltage class, line length, and design features. It may be represented by the active and inductive resistances parameters and the active and capacitive conductivity parameters. The mathematical model of any high-voltage transmission line (HVTL) may be presented as equations of the steady-state or the transient conditions. Moreover, recording of equations can be a simplified form without taking into account some mode parameters (fault resistance in fault point, the load current, and others) or in a specified form.

The $110 \mathrm{kV}$ to $330 \mathrm{kV}$ transmission lines, up to $300 \mathrm{~km}$ long, use the line model, based on the steady-state equations with the resistance $R$ and impedance $X$ parameters to fault location. For such model, the control bus voltage equation during a steadystate fault condition has the following form:

$$
\dot{U}=R \cdot \dot{I}+j X \cdot \dot{I} \text {. }
$$

The system may be solved relatively the unknown parameters by the following calculated expressions for each moment of sampling:

$$
R=\frac{U_{\mathrm{c}} \cdot I_{\mathrm{c}}+U_{\mathrm{s}} \cdot I_{\mathrm{s}}}{I_{\mathrm{c}}{ }^{2}+I_{\mathrm{s}}^{2}} ; X=\frac{U_{\mathrm{s}} \cdot I_{\mathrm{c}}-U_{\mathrm{c}} \cdot I_{\mathrm{s}}}{I_{\mathrm{c}}{ }^{2}+I_{\mathrm{s}}{ }^{2}},
$$

where

$U_{\mathrm{c}}, U_{\mathrm{s}}, I_{\mathrm{c}}, I_{\mathrm{s}}$ are the orthogonal components of the base harmonic current and voltage, obtained during the digital filtering of the input signals. Usually, the algorithm of Discrete Fourier Transform is used. For example, each moment of current sampling the orthogonal components are calculated by expressions:

$$
\left.I_{\mathrm{c}}(n, T)=\sum_{l=0}^{N} g_{\mathrm{c}, l} \cdot i[(n-l) T]\right), I_{\mathrm{s}}(n, T)=\sum_{l=0}^{N} g_{\mathrm{s}, l} \cdot i[(n-l) T],
$$

where $g_{\mathrm{s}, l}, g_{\mathrm{c}, l}$ - coefficients of orthogonal digital filter;

$$
g_{\mathrm{s}, l}=\frac{\omega T}{2 \pi} \sin \omega l T ; \quad g_{\mathrm{c}, l}=\frac{\omega T}{2 \pi} \cos \omega l T ;
$$

$N$ is the number of sampling intervals, usually for one period of industrial frequency;

$$
u[(n-l) T], i[(n-l) T] \text { are the voltage and current samples; }
$$

\footnotetext{
* Corresponding author.

E-mail address: loman@eef.rtu.lv
} 


$$
\omega=2 \pi F_{0}, F_{0}=50 \mathrm{~Hz} \text {; }
$$

$T$ is the time interval between two samples.

The algorithm of the digital filtration of current and voltage in order to represent the vector module in a complex form in $n$ moment of analogue-to-digital conversion:

$I(n, T)=I_{\mathrm{c}}(n, T)+j I_{\mathrm{s}}(n, T)$,

$U(n, T)=U_{\mathrm{c}}(n, T)+j U_{\mathrm{s}}(n, T)$,

where

$i(n, T), u(n, T)$ - are discrete current and voltage signals in $n$ interval of analogue-to-digital conversion.

The phase impedance during the dynamic operation is calculated according to the following algorithm:

$$
\begin{aligned}
& X(n, T)=\operatorname{Im}\left[\frac{\dot{U}_{\mathrm{ph}}(n, T)}{\dot{I}_{\mathrm{ph}}(n, T)+\dot{k} \dot{I}_{0}(n, T)}\right] ; \\
& R(n, T)=\operatorname{Re}\left[\frac{\dot{U}_{\mathrm{ph}}(n, T)}{\dot{I}_{\mathrm{ph}}(n, T)+\dot{\mathrm{I}}_{0}(n, T)}\right],
\end{aligned}
$$

where $\dot{k}=\left(\dot{Z}_{L 1}-\dot{Z}_{L 0}\right) / \dot{Z}_{L 1}$;

$\dot{Z}_{L 1}, \dot{Z}_{L 0}$ - sequence impedance of the line per unit length;

$\dot{U}_{\mathrm{ph}}(n, T)$ - phase voltage in $n T$-discrete moment;

$\dot{I}_{\mathrm{ph}}(n, T), \dot{I}_{0}(n, T)$ are vectors of the phase and zero sequence current in $n, T$-discrete moment.

The transition process in the simplified line model with concentrated parameters of resistance $R$ and inductance $L$ is described by the differential equation, and for analog systems it may be shown as:

$$
u(t)=R \cdot i(t)+L \cdot \frac{d i}{d t},
$$

where $L=X / \omega$.

The equation (3) complex form for discrete systems is the following:

$$
\dot{U}(n, T)=R \cdot \dot{I}(n, T)+\frac{X}{\omega_{0}} \cdot \frac{\Delta \dot{I}(n, T)}{\Delta T},
$$

where $\omega_{0}=314 \mathrm{rad} / \mathrm{s} ; T$ is sampling time

$\Delta \dot{I}(n, T)$ is the difference between the instantaneous values of currents;

$\Delta T$ is the time interval between two samples.

The use of the differential equation (4) provides the removal of the exponential component of the transient process from the input signal. The solution of equations with respect to $R$ and $X$ takes the form:

$$
\begin{gathered}
R=\frac{U_{\mathrm{c}} \cdot \Delta I_{\mathrm{c}}+U_{\mathrm{s}} \cdot \Delta I_{\mathrm{s}}}{I_{\mathrm{c}} \cdot \Delta I_{\mathrm{c}}+I_{\mathrm{s}} \cdot \Delta I_{\mathrm{s}}} \\
X=\omega_{0} \cdot \Delta t \cdot \frac{U_{\mathrm{s}} \cdot \Delta I_{\mathrm{c}}-U_{\mathrm{c}} \cdot \Delta I_{\mathrm{s}}}{\Delta I_{\mathrm{c}} \cdot I_{\mathrm{c}}+\Delta I_{\mathrm{s}} \cdot I_{\mathrm{s}}}
\end{gathered}
$$

where

$\Delta I_{\mathrm{c}}$ and $\Delta I_{\mathrm{s}}$ are the difference between the instantaneous values of orthogonal components of the base harmonic vector, obtained during the digital filtering of the input signals by using DFT algorithm.

The transition process in the simplified line model with the concentrated parameters of resistance $R$ and inductance $L$ and the active $(G)$ and capacitive $(j w C)$ conductivity is described by the following differential equation:

$$
\begin{gathered}
U=R \cdot i+L \cdot \frac{d i}{d t}-G \cdot R \cdot U-(R \cdot C+L \cdot G) \frac{d u}{d t}-L \cdot C \cdot \frac{d^{2} u}{d t^{2}} ; \\
\text { if } G=0, \\
\text { then } U=R \cdot i+L \cdot \frac{d i}{d t}-R \cdot C \cdot \frac{d u}{d t}-L \cdot C \cdot \frac{d^{2} u}{d t^{2}} .
\end{gathered}
$$

\section{Classic Algorithm of the Earth Fault DMU}

The classic algorithm of the earth fault DMU is synthesized by the simplified line model represented with the active resistance $R$ and inductance $L$ without taking into account additional factors. Then, the node-voltage equation for controllable bus during the earth fault in a line is as follows:

$$
\dot{U}_{\mathrm{ph}}=\dot{Z}_{\mathrm{F} 1} \cdot\left(\dot{I}_{\mathrm{ph}}+\dot{K}_{\mathrm{N}} \cdot \dot{I}_{\mathrm{E}}\right) \text {, }
$$

where

$$
\dot{K}_{\mathrm{N}}=\frac{\dot{Z}_{\mathrm{L} 0}-\dot{Z}_{\mathrm{L} 1}}{3 \cdot \dot{Z}_{\mathrm{L} 1}} ;
$$

$\dot{Z}_{\mathrm{L} 1}, \dot{Z}_{\mathrm{L} 0}$ are respectively the positive and zero sequence impedance of the line;

$\dot{Z}_{\mathrm{F} 1}$ is the positive sequence impedance from the relay location and fault point; $\dot{U}_{\mathrm{ph}}$ and $\dot{I}_{\mathrm{ph}}$ are the phase voltage and current. Then

$$
\dot{U}_{\mathrm{ph}}=\dot{U}_{\mathrm{L} 1}+\dot{U}_{\mathrm{L} 2}+\dot{U}_{\mathrm{L} 0}, \dot{I}_{\mathrm{ph}}=\dot{I}_{\mathrm{L} 1}+\dot{I}_{\mathrm{L} 2}+\dot{I}_{\mathrm{L} 0},
$$

$\dot{I}_{\mathrm{L} 1}, \dot{I}_{\mathrm{L} 2}, \dot{I}_{\mathrm{L} 0} ; \dot{U}_{\mathrm{L} 1}, \dot{U}_{\mathrm{L} 2}, \dot{U}_{\mathrm{L} 0}$ are respectively positive, negative, and zero sequence currents and voltages;

$\dot{I}_{\mathrm{E}}$ is the earth current, then $\dot{I}_{\mathrm{E}}=3 \dot{I}_{\mathrm{L} 0}$.

The solution of equation (6) with respect to the unknown impedance is in the following form [5]-[11]:

$$
\dot{Z}_{\mathrm{F} 1}=\frac{\dot{U}_{\mathrm{ph}}}{\dot{I}_{\mathrm{ph}}+\dot{K}_{\mathrm{N}} \cdot \dot{I}_{\mathrm{E}}} .
$$

No matching simulated conditions underlying the synthesis of classical DMU algorithms and the actual conditions of the emergency regime lead to significant errors in the calculation 
of the positive sequence line impedance vector, especially for large values of transient resistance at the fault point.

The algorithms [6]-[12] based on the protected object specified model take into account the transient resistance in the fault location and the changes in the scheme and regime of a monitored object. It is especially important in complex configuration networks and during earth fault with large values of transient resistance at the fault location.

\section{Mathematical Model of a Protected ObJect}

The node-voltage equations actually use the earth fault DMU algorithms. Then the controlled bus voltage vectors can be represented as a function of three vectors:

$$
\dot{U}_{\text {bus }}=f\left(\bar{\Pi}_{\mathrm{m}}, \bar{\Pi}_{\text {const }}, \bar{\Pi}_{\mathrm{fl}}\right) \text {, }
$$

where

$\bar{\Pi}_{\mathrm{m}, i}$ is the vector of control parameters:

$$
\bar{\Pi}_{\mathrm{m}, i}=f\left(\dot{I}_{\mathrm{ph}}, \dot{I}_{0}, \dot{I}_{1}, \dot{I}_{2}, \dot{U}_{1}, \dot{U}_{0}, \dot{U}_{2}, \dot{U}_{\mathrm{ph}}\right),
$$

$\dot{I}_{\mathrm{ph}}, \dot{I}_{1}, \dot{I}_{2}, \dot{I}_{0}$ are the phase, positive, negative, and zero sequence current in line protection;

$\dot{U}_{1}, \dot{U}_{0}, \dot{U}_{2}, \dot{U}_{\mathrm{ph}}$ are the positive, negative, and zero sequence and phase voltage in protection;

$\bar{\Pi}_{\text {const }}$ is known parameters vector:

$$
\bar{\Pi}_{\text {const }}=f\left(\dot{Z}_{\mathrm{s}}, L, \dot{Z}_{\mathrm{L} 1}, \dot{Z}_{\mathrm{L} 0}, \dot{U}_{\mathrm{EMF}}\right),
$$

$\dot{Z}_{\mathrm{L} 1}, \dot{Z}_{\mathrm{L} 0}$ are respectively the positive and zero sequence impedance of the line;

$L$ line length; $\dot{Z}_{\text {s }}$ is the power system impedance;

$\dot{U}_{\text {EMF }}$ - constant voltage of generators EMF.

$\bar{\Pi}_{\mathrm{fl}}$ is unknown parameters vector

$$
\bar{\Pi}_{\mathrm{fl}}=f\left(\dot{Z}_{\text {bus_fault }}, \dot{S}, R_{F}\right) ;
$$

$\dot{Z}_{\text {bus fault }}$ - the positive sequence line impedance between control bus and fault location $F$;

$\dot{S}$ - vectors of mode parameters;

$R_{\mathrm{F}}$ is fault resistance in the fault location.

Definition of the vector $\bar{\Pi}_{\mathrm{fl}}$ of unknown parameters by the protected object model equation taking into account all parameters of the emergency conditions is a difficult task because of the non-linear function of the variables. Therefore, there are many models corresponding to the various simplifications of the vector of unknown parameters. The nodevoltage equations considering the transition resistance in the fault location has the form:

$$
\dot{U}_{\text {bus }}=\dot{U}_{\text {bus_fault }} \cdot \dot{I}_{\mathrm{m}}+\dot{I}_{\text {fault }} \cdot R_{\text {fault }},
$$

where

$\dot{Z}_{\text {bus fault }}$ is the positive sequence impedance from relay location to the fault point;
$\dot{I}_{\mathrm{m}}$ is the measuring current of DMU;

$\dot{I}_{\text {fault }}=f\left(\dot{Z}_{\text {bus_fault }} ; \dot{S}\right)$ is the earth current in the fault point;

$\dot{S}$ is the vector of network parameters.

The earth fault DMU monitors the condition of the protected object by the values of the phase and zero sequence. Currents monitors the condition of the protected object by the values of the bus voltage in the protection relay location. Let us consider the line between substations $\mathrm{A}$ and $\mathrm{B}$, and the distance protection is placed on the substation $\mathrm{A}$.

The node voltages at the ends of the line during the earth fault mode may be calculated by expressions:

$$
\begin{gathered}
\dot{U}_{\mathrm{A}}=\dot{Z}_{\mathrm{AF} 1} \cdot\left(\dot{I}_{\mathrm{A}}+\dot{K}_{\mathrm{N}} \cdot \dot{I}_{\mathrm{A} 0}\right)+\dot{I}_{\mathrm{EF}} \cdot R_{\mathrm{F}}, \\
\dot{U}_{\mathrm{B}}=\left(\dot{Z}_{\mathrm{L} 1}-\dot{Z}_{\mathrm{AF} 1}\right) \cdot\left(\dot{I}_{\mathrm{B}}+\dot{K}_{\mathrm{N}} \cdot \dot{I}_{\mathrm{B} 0}\right)+\dot{I}_{\mathrm{EF}} \cdot R_{\mathrm{F}},
\end{gathered}
$$

where

$$
\begin{gathered}
\dot{K}_{\mathrm{N}}=\frac{\dot{Z}_{\mathrm{L} 0}-\dot{Z}_{\mathrm{L} 1}}{3 \cdot \dot{Z}_{\mathrm{L} 1}} \\
\dot{I}_{\mathrm{B}, \mathrm{bus}}=\dot{I}_{\mathrm{B} 1}+\dot{I}_{\mathrm{B} 2}+\dot{I}_{\mathrm{B} 0} ; \\
\dot{I}_{\mathrm{B} 1}=\dot{I}_{\mathrm{F} 1}-\dot{I}_{\mathrm{A} 1} ; \\
\dot{I}_{\mathrm{B} 2}=\dot{I}_{\mathrm{F} 2}-\dot{I}_{\mathrm{A} 2} ; \\
\dot{I}_{\mathrm{B} 0}=\dot{I}_{\mathrm{F} 0}-\dot{I}_{\mathrm{A} 0} \\
\dot{I}_{\mathrm{EF}}=3 \cdot \dot{I}_{\mathrm{F} 0} ; \\
\dot{I}_{\mathrm{F} 0}=\dot{K}_{\mathrm{cur}} \cdot \dot{I}_{\mathrm{A} 0} ; \\
\dot{K}_{\mathrm{cur}}=\frac{\dot{Z}_{\mathrm{B} 0}+\left(\dot{Z}_{\mathrm{L} 0}-\dot{Z}_{\mathrm{AF} 0}\right)}{\dot{Z}_{\mathrm{B} 0}+\dot{Z}_{\mathrm{L} 0}+\dot{Z}_{\mathrm{A} 0}}
\end{gathered}
$$

$\dot{Z}_{\mathrm{L} 1}, \dot{Z}_{\mathrm{L} 0}$ are respectively the positive and zero sequence impedance of the line per unit length;

$\dot{Z}_{\mathrm{B} 1}, \dot{Z}_{\mathrm{B} 0}, \dot{Z}_{\mathrm{A} 1}, \dot{Z}_{\mathrm{A} 0}$ are the positive and zero sequence impedances of the equivalent systems $\mathrm{A}$ and $\mathrm{B}$;

$\dot{Z}_{\mathrm{AF} 1}$ is the positive sequence impedance from relay location and fault point;

$R_{\mathrm{F}}$ - is fault resistance in the fault point;

$\dot{I}_{\mathrm{A} 1}, \dot{I}_{\mathrm{A} 2}, \dot{I}_{\mathrm{A} 0}$ are the positive, negative and zero sequence currents; $\dot{U}_{\mathrm{A}}, \dot{U}_{\mathrm{B}}$ are the phase voltage;

$\dot{I}_{\mathrm{A}}, \dot{I}_{\mathrm{A} 0}$ are respectively the phase and zero sequence currents.

On the other hand, the bus voltage $\dot{U}_{\mathrm{A}}$ at the protection relay installation is known by the DMU measurements, but the bus voltage $\dot{U}_{\mathrm{B}}$ can be calculated by the B system parameters:

$$
\dot{U}_{\mathrm{B}, \text { bus }}=\dot{E}_{\mathrm{B}}-2 \cdot \dot{Z}_{\mathrm{B} 1} \cdot \dot{I}_{\mathrm{B} 1}-\dot{I}_{\mathrm{B} 0} \cdot \dot{Z}_{\mathrm{B} 0},
$$

where $\dot{E}_{\mathrm{B}}$ is the EMF of B system.

Then, the nodes voltage balance equations can be written as follows: 


$$
\left.\begin{array}{l}
\dot{U}_{\mathrm{b}, \mathrm{A}}=\dot{U}_{\mathrm{A}, \mathrm{m}}-\dot{U}_{\mathrm{A}} \\
\dot{U}_{\mathrm{b}, \mathrm{B}}=\dot{U}_{\mathrm{B}, \mathrm{bus}}-\dot{U}_{\mathrm{B}}
\end{array}\right\},
$$

where

$\dot{U}_{\mathrm{A}, \mathrm{m}}$ is the measured value of the A bus voltage;

$\dot{U}_{\mathrm{A}}, \dot{U}_{\mathrm{B}}$ are the calculated values of the $\mathrm{A}$ and $\mathrm{B}$ bus voltage;

$\dot{U}_{\mathrm{b}, \mathrm{A}}, \dot{U}_{\mathrm{b}, \mathrm{B}}$ are the voltage balance vectors of the $\mathrm{A}$ and $\mathrm{B}$ nodes.

The equations system (13) use as an objective function in optimization tasks for searching the effective values of unknown variables and positive sequence impedance from relay location to fault point [12].

\section{DMU ALGORITHMS FOR COMPLEX CONFIGURATION NetWork PROTECTION}

Multicircuits and multiend lines are considered as complex configuration networks. Using the classical algorithms for DMU operation in such networks is not always effective. In order to improve the DMU efficiency, the algorithms based on the protected object model with taking into account the particular network configuration are evaluated [6]-[11]

When a ground fault in parallel lines, the undesirable factor affecting the relay operation is the mutual EMF induced by the zero sequence currents of the parallel circuit. Its value depends not only on the current, but also on the operation mode of parallel lines and fault location. The most modern microprocessor protection devices provide the ability to install different groups of settings and compensation factors for the operation of different modes of parallel lines, as well as protection zones for considering the mutual EMF influence.

In the absence of such possibility in the devices, it is necessary to determine the optimal value of the compensation factor for each step of the DMU. In most cases, the multicircuit lines have two parallel lines. Depending on the position of the switches on the parallel lines, the mutual EMF influence is changed, so the zero sequence impedance is changed too. The following operation conditions can be considered.

1. Normal condition, when both parallel lines are in operation.

In this mode, the zero-sequence equivalent impedance during the earth fault on the bus of line's opposite end is calculated by the expression:

$$
\dot{Z}_{\mathrm{E} 0}=\dot{Z}_{\mathrm{L} 0}+\dot{Z}_{\mathrm{M} 0},
$$

where $\dot{Z}_{\mathrm{L} 0}, \dot{Z}_{\mathrm{M} 0}$ are the zero sequence and mutual zero sequence line impedance.

2. Operation condition when one of parallel lines is switched off and grounded on both ends.

In this mode, the zero-sequence equivalent impedance of parallel lines during the earth fault on the bus of line opposite end is calculated by the expression:

$$
\dot{Z}_{\mathrm{E} 0}=\frac{\dot{Z}_{\mathrm{L} 0}^{2}-\dot{Z}_{\mathrm{M} 0}^{2}}{\dot{Z}_{\mathrm{L} 0}} .
$$

3. Operation condition when one of parallel lines is switched off and without ground on both ends.

In this mode, the zero-sequence equivalent impedance of parallel lines during the earth fault on the bus of line opposite end is calculated by the expression:

$$
\dot{Z}_{\mathrm{E} 0}=\dot{Z}_{\mathrm{L} 0} .
$$

The lengthening or shortening of the protected area due to the influence of the mutual zero-sequence impedance is necessary to compensate by using the appropriating models. Because of the wide range of the zero sequence equivalent impedance values corresponding to the various states of the line breakers to ensure an effective operation of the protection relay in all modes is very difficult. The most widely used compensation methods are as follow.

The introduction of an additional compensation factor of the mutual zero sequence current in the impedance calculating expression as follows [5]:

$$
\dot{Z}_{\mathrm{m} 1}=\frac{\dot{U}_{\mathrm{ph}}}{\dot{I}_{\mathrm{ph}}+\dot{K}_{\mathrm{N}} \cdot \dot{I}_{\mathrm{E}}+\dot{K}_{\mathrm{M} 0} \cdot \dot{I}_{\mathrm{Ep}}},
$$

where

$\dot{K}_{\mathrm{M} 0}=\frac{\dot{Z}_{\mathrm{M} 0}}{3 \cdot \dot{Z}_{\mathrm{L} 1}}$ is the compensation factor of zero-sequence mutual coupling;

$\dot{I}_{\text {Ep }}$ is the earth current of the parallel operating line;

$\dot{Z}_{\mathrm{M} 0}$ is zero sequence mutual impedance;

$\dot{K}_{\mathrm{N}}=\frac{\dot{Z}_{\mathrm{L} 0}-\dot{Z}_{\mathrm{L} 1}}{3 \cdot \dot{Z}_{\mathrm{L} 1}}$ is the earth compensation factor;

$\dot{I}_{\mathrm{E}}=3 \cdot \dot{I}_{0}$ is the earth current;

$\dot{I}_{0}$ is zero sequence currents;

$\dot{Z}_{\mathrm{L} 0}, \dot{Z}_{\mathrm{L} 1}$ are respectively the positive and zero sequence impedance of the line per unit length;

$\dot{U}_{\mathrm{ph}}, \dot{I}_{\mathrm{ph}}$ are the phase voltage and current at the relay location in the faulted phase.

The using the optimal compensation coefficient in classical algorithm DMU during the earth faults

$$
\dot{Z}_{\mathrm{m} 1}=\frac{\dot{U}_{\mathrm{ph}}}{\dot{I}_{\mathrm{ph}}+\dot{K}_{\mathrm{NE}} \cdot \dot{I}_{\mathrm{E}}},
$$

where $\quad \dot{K}_{\mathrm{NE}}=\frac{\dot{Z}_{\mathrm{E} 0}-\dot{Z}_{\mathrm{L} 1}}{3 \cdot \dot{Z}_{\mathrm{L} 1}}$.

$\dot{Z}_{\mathrm{E} 0}$ is the equivalent zero sequence impedance, determined from the design mode.

The calculated expression (17) is common for all modes, but the calculated expression (18) can be used only in adaptive protection, when the compensation factor is different in different modes of the network configuration. 


\section{CONCLUSION}

- PDMU algorithms are synthesized on the protected object model base. The efficiency of the algorithms depends on the coincidence of the model with the actual conditions of emergency operation.

- To increase the effectiveness of DP operation during the transitional conditions, the PDMU algorithms must be synthesized on the basis of the transitional processes equations.

- To increase the effectiveness of DP in complex configuration networks, the adaptive PDMU algorithm, where the network configuration is considered, is used.

\section{REFERENCES}

[1] M. Begovic, D. Novosel and M. Milisavljevic, "Development Trends in Power System Protection and Control," in Proc. of 32nd Hawaii Int. Conf. on System Sciences, 1999, pp. 1-8.

[2] A. Utans, A. Sauhats and E. Bieḷa-Dailidoviča, "Wide-Area Measurments Based Out-of-Step Protection System," in Proc. 56th Int. Conf. on Power and Electricity of RTUCON-2015, Riga, Oct. 14, 2015, pp. 11-15. http://dx.doi.org/10.1109/rtucon.2015.7343113

[3] S. Kovalenko, A. Sauhats, I. Zicmane and A. Utans, "New Methods and Approaches for Monitoring and Control of Complex Electrical Power System Stability," in Proc. 16th IEEE EEEIC 2016, Italy, Florence, June 7-10, 2016, pp. 270-275.

[4] D. J. Rummer and M. A. Kezunovic, "Survey and Classification of the Digital Computer Relaying Literature," IEEE Power Eng. Soc., Paper of Summer Meeting, Vancouver, Canada, 1979, pp. 417 -7/1-7/7.
[5] T. Loman, A. Sauhatas and A. Voinovska, "Optimization of Parameters and Algorithms of Distance Protection," in IV Int. Symp. MMwEE'98, Zacopane, Poland, Oct. 7-10, 1998, pp. 1-6.

[6] ABB Relays REL 511, Line distance protection terminal, User's Guide, ABB Network Control \& Protection, Sweden, 1995.

[7] J. Izykowski, E. Rosolowski, M. Saha, "Adaptive Digital Distance Algorithm for Parallel Transmission Lines," in IEEE Bologna Power Tech Conf., Bologna, Italy, June 23-26, 2003. http://dx.doi.org/10.1109/ptc.2003.1304598

[8] M. Bozek, et al., "Distance Protection Algorithm for Double-circuit Transmission Line with Fault Resistance Compensation," in 17th Power Systems Computation Conf., Stockholm, Sweden, Aug. 22-26, 2011.

[9] P. Mazniewski and J. Izykowski, "ATR-EMTR Investigation of Adaptive Distance Protection for Transmissions Lines with Series Compensation," Modern Electric Power Systems, Wroclaw, Poland, paper P28, 2010.

[10] S. G. Srivani, C. R. Atla and K. P. Vittal, "Comparative Evaluation of Adaptive and Conventional Distance Relay for Parallel Transmission Line with Mutual Coupling," Int. J. of Electrical, Computer, Energetic, Electronic and Commun. Eng., vol. 2, no.6, pp. 1168-1174, 2008

[11] T. Loman, A. Voinovska and R. Koemecs, "Estimating the effectiveness of distance measuring units operation in the parallel lines," in The 6th Int. Conf. EMES'01, Oradea, Romania, May 24-26, 2001, pp. 361-367.

[12] T. Loman, V. Belugina and R. Koemecs, "The Distance Measuring Units Algorithm Synthesis On The Basis Of The Earth Fault Specified Model," J. of Comput. Science and Control Systems, Oradea, Romania, vol. 2, part 1, pp. 107-110, 2009.

[13] T. Loman, V. Belugina, "The Effectiveness Increasing For Distance Protection," in 13th Int. Conf. APE'07, Jurata, Poland, June 11-13, 2007. 